\title{
Aplicación de lógica difusa en el proceso de shot peening del aluminio 2024-T351
}

\author{
Alicia Guadalupe Lazcano Herrera ${ }^{1}$, Sandra Silvia. Roblero Aguilar ${ }^{1},{ }^{2}$, \\ José Solis Romero ${ }^{1}$, Héctor Rafael Orozco Aguirre ${ }^{2}$, \\ Víctor Augusto Castellanos Escamilla ${ }^{1}$ \\ ${ }^{1}$ SEP/SES/TecNM/Instituto Tecnológico de Tlalnepantla, Estado de México, \\ México \\ ${ }^{2}$ Centro Universitario UAEM Valle de México, Estado de México, \\ México
}

\{alicia_gpe_hg, ssrauaemex $\} @$ hotmail.com, \{jsolis,vcastellanos\}@ittla.edu.mx, rafilla.orozco@gmail.com

\begin{abstract}
Resumen. El proceso de granallado o shot peening se emplea rutinariamente para incrementar la resistencia a la fatiga de componentes y estructuras. Los efectos que se producen en la superficie del material tratado son esfuerzos compresivos residuales, rugosidad superficial y endurecimiento por deformación, los cuales dependen de la correcta elección de los parámetros de procesamiento. En esta investigación se aplica el método de lógica difusa para determinar la mejor combinación de factores de control que inciden en las respuestas multi-objetivo de una aleación de aluminio 2024-T351 (AA) tratada con shot peening. Los factores de control de entrada son el tipo de bolilla (shot), la cobertura y el ángulo de incidencia. Para fines estadísticos, los parámetros experimentales se trabajaron utilizando un arreglo fraccional ortogonal L16. Las tres propiedades que se determinaron en forma experimental directamente sobre AA tratada son las entradas para el sistema de inferencia difuso y la salida es el índice de respuesta (IR). Se realizó una comparación del IR entre las condiciones de tratamiento inicial con las óptimas, resultando una mejora en la resistencia a la fatiga.
\end{abstract}

Palabras clave: Lógica difusa, sistema de inferencia difuso, shot peening/Granallado

\section{Application of Fuzzy Logic in the Process of Shot Peening of Aluminium 2024-T351}

Abstract. Shot peening is widely used to enhance the fatigue properties of components and structures. Compressive residual stresses, surface roughness, 


\begin{abstract}
and work hardening are the immediate effects induced on the surface/subsurface layer of the treated material, which depend on the correct choice of the peening process parameters. This research aims to apply the fuzzy logic method for determining the best optimal selection of the control factors that directly influence the multi-objective response properties of a peened 2024-T351 aluminium alloy (AA). The input parameters taken into consideration are shot, coverage and incidence angle. For statistical purposes, the experimental parameters were put in place with a $\mathrm{L}_{16}$ orthogonal fractional array. The three induced properties extracted experimentally from the treated AA are fed as inputs to fuzzy inference system and output withdrew is the response index (IR). A comparison of the IR between the initial and optimal peening conditions shows an improvement in fatigue resistance.
\end{abstract}

Keywords: Fuzzy logic, fuzzy inference system, shot peening.

\title{
1. Introducción
}

Los componentes utilizados tanto en la industria aeroespacial como en la industria automotriz a menudo están sujetos a condiciones de carga fluctuantes o cíclicas, dando origen a la fatiga de componentes. Las grietas por fatiga se originan normalmente desde la superficie de la parte debido, entre otros, a defectos contenidos en el material [1]. Cuando la carga dinámica continúa, la grieta crece hasta que alcanza una longitud tal que ninguna barrera le podrá disminuir su velocidad de crecimiento o detener. Sin embargo, cuando la grieta creciente se obstruye en su camino por diversas barreras muy compactas, como ocurriría en microestructuras que contienen granos pequeños, entonces el crecimiento de la grieta disminuye la velocidad de crecimiento debido a que su trayectoria se desvía lo que resulta en un incremento en la resistencia a la fatiga. Es por ello que la resistencia a la fatiga usualmente se cuantifica como la resistencia a la propagación de grietas ofrecida por el material [2].

Para acondicionar la superficie en forma tal que permita detener o disminuir la velocidad de propagación de grietas, el proceso de granallado o shot peening (SP) es una alternativa sencilla y económica, en donde se introducen muchas y variadas formas de barreras de textura o distorsión microestructural en la superficie y capas subsuperficiales. En este proceso, esencialmente, bolillas esféricas conocidas como shots, que están hechas de acero al carbón, hierro, acero inoxidable, vidrio o trozos redondeados de cerámico, se proyectan en forma deliberada para golpear la superficie de un componente metálico. Las bolillas se aceleran ya sea por medio de aire comprimido o por fuerzas centrífugas. Las velocidades al impacto son suficientes para que se origine una indentación (huella) en la superficie del material tratado, en donde el régimen que domina es completamente plástico. Precisamente ese régimen plástico da origen a un estado de esfuerzos residuales compresivos y un endurecimiento por deformación en la sub-superficie, los cuales forman parte de esas barreras que inherentemente obstruyen el libre movimiento de las grietas.

En el proceso de SP la magnitud de los parámetros de procesamiento, así como todos los efectos inducidos deben controlarse a fin de lograr un beneficio en términos de resistencia a la fatiga y evitar o reducir la introducción de daño severo (por ejemplo, 
iniciación de grieta causada por una excesiva rugosidad) [3, 4]. Por lo tanto, el desarrollo de un método rápido y eficiente para optimizar el proceso es una importante área de estudio para realizar investigación. Bajo este contexto, el diseño de experimentos estadístico (DoE por sus siglas en inglés) está considerado como una herramienta consolidada para resolver problemas relacionados con la elección de los parámetros óptimos de procesamiento [5]. Unal [6] utilizó el DoE para la optimización de los parámetros en SP sobre probetas estandarizadas llamadas tiras Almen.

La optimización se realizó extrayendo los niveles óptimos de cada propiedad y posteriormente se compilaron los parámetros de procesamiento. Por otro lado, George [7] aplicó la metodología DoE con Taguchi para lograr la optimización de parámetros críticos, así como establecer el orden de predominancia, utilizando el análisis de varianza (ANOVA) para finalmente predecir un arreglo óptimo de cada parámetro. En breve, el DoE emplea diseños ortogonales para examinar las características de calidad por medio de un número reducido de experimentos. Sin embargo, esta técnica está limitada cuando se trata del tratamiento de problemas multi-respuesta, en otras palabras, el nivel óptimo de los parámetros de procesamiento es verdadero para la optimización de una respuesta individual del proceso.

Para contrarrestar lo anterior, la técnica de lógica difusa [8] tiene la capacidad de trabajar con diversas entradas, como por ejemplo, características funcionales de procesos, para eventualmente convertir el comportamiento múltiple en un solo índice multi-respuesta (IR) [9]. El procedimiento de cómputo suave como la lógica difusa está ganando una aceptación progresiva en una variedad de situaciones ingenieriles [10-12], en virtud de su utilidad cuando no se encuentra disponible información matemática exacta. En comparación con otros métodos de la inteligencia artificial, el desarrollo de la lógica difusa es moderadamente más sencillo y no necesita de mucho software ni demasiado hardware. Sin embargo, hasta donde los autores tienen conocimiento, no se reportan trabajos dedicados a la aplicación de esta metodología como una estrategia para optimizar el proceso de SP con la intención de incrementar la resistencia a la fatiga de materiales metálicos.

En el presente trabajo, se emplea un mecanismo de inferencia difuso para determinar los parámetros óptimos de procesamiento en el tratamiento superficial del aluminio 2024-T351, utilizando las respuestas experimentalmente determinadas como son los esfuerzos residuales, el endurecimiento por deformación y la rugosidad superficial en términos de concentración de esfuerzos.

\section{Generalidades de la lógica difusa}

La lógica difusa (LD) está relacionada y fundamentada en la teoría de los conjuntos difusos, en la cual, el grado de pertenencia de un elemento a un conjunto está determinado por una función de pertenencia (FP) [13-15] que puede adoptar valores reales comprendidos en el intervalo [0,1],. De esta manera, mientras que en el marco de la lógica clásica un parámetro tiene pertinencia o no, dándole un valor de 1 si es pertinente y 0 en caso contrario; En la LD, se obtiene un nivel de cumplimiento de la 


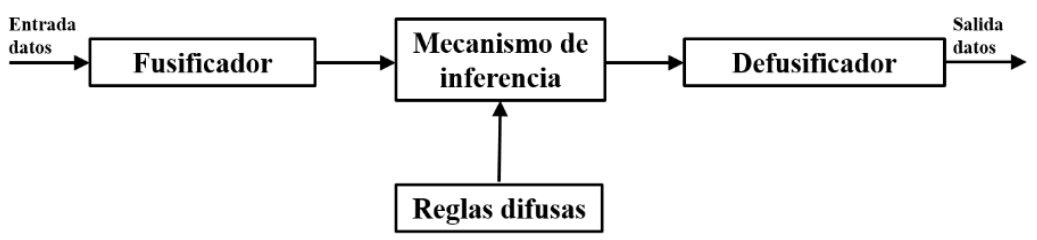

Fig. 1. Esquema general de funcionamiento de un sistema de inferencia difuso.

pertinencia, es decir, entre más cercano a cero, será menos pertinente y cuando sea más cercano a 1 será más pertinente.

Las fases que completan el montaje de un sistema de inferencia difuso Mandani [15, 16] se muestran en la Fig. 1. Textualmente se describen como sigue:

- Fase 1: se basa en un proceso donde las variables tienen un grado de incertidumbre metalingüístico. Es decir, el rango de valores de cada variable puede clasificarse por conjuntos difusos, originando el universo del discurso. Con ello, los valores pasan a un proceso de fusificación que los categoriza en un nivel de pertenencia entre 0 y 1 que pertenece a un conjunto difuso. Los conjuntos difusos son caracterizados mediante funciones de pertenencia, las cuales están sintonizadas al punto de operación adecuado para el funcionamiento del sistema, es decir, las reglas de inferencia que serán empleadas

- Fase 2: se proponen reglas lingǘ́sticas conocidas como de inferencia. Con esto, el grado de pertenencia de cada una de las variables se evalúa en un subconjunto de estas reglas.

Fase 3: aquí se determinan los valores óptimos de salida, mediante un mecanismo conocido como defusificación, el cual consiste en pasar el grado de pertenencia, proveniente de la consecuencia de la regla de inferencia activada, a un valor nítido o real, con el fin de obtener un valor cuantificable.

\section{Caso de estudio: aplicación de lógica difusa en la selección óptima de parámetros del proceso de shot peening}

El desarrollo del presente estudio se llevó a cabo bajo la consideración de las siguientes etapas: recopilación de datos, fusificación, generación de reglas y la aplicación del sistema de inferencia difuso (FIS, por sus siglas en inglés).

\subsection{Recopilación de datos}

En general, del proceso SP previamente descrito en la introducción, la información que se utiliza en el presente análisis se puede clasificar como (i) los factores de control de procesamiento y (ii) las propiedades de respuesta o efectos inducidos. Los factores de control que se eligieron son el tipo/tamaño de bolilla o shot (1), la cobertura en porcentaje (2) y el ángulo de incidencia o impacto (3). Por su parte, las propiedades de 

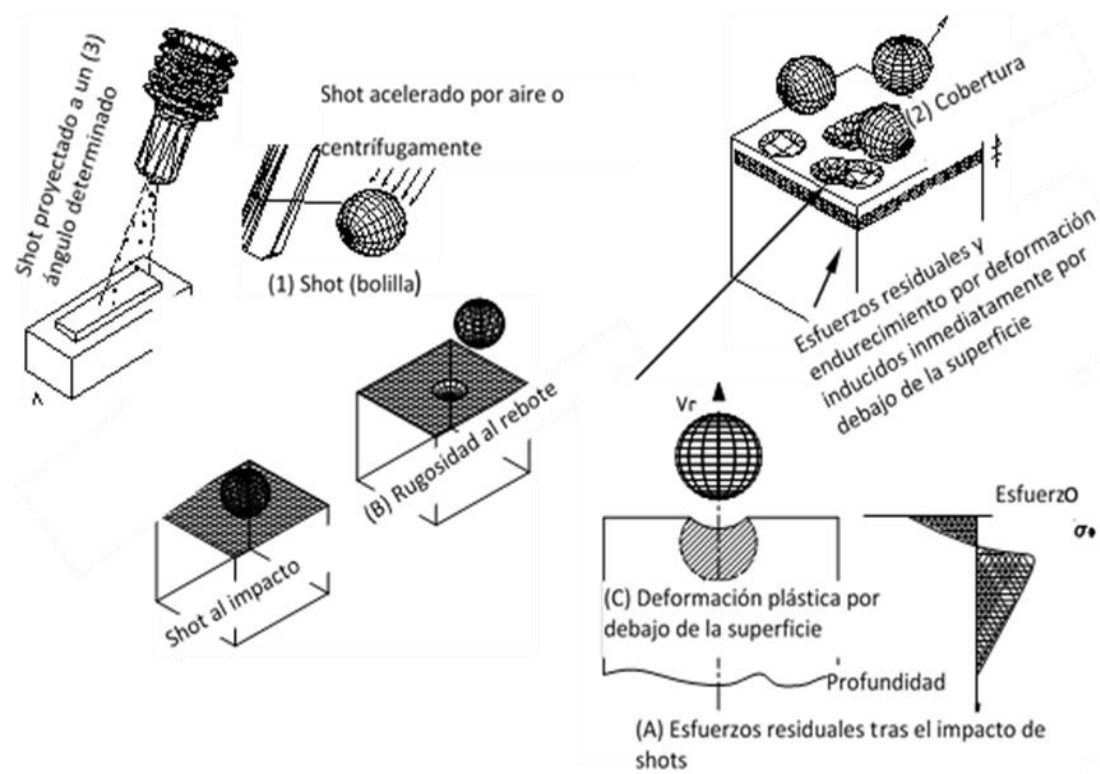

Fig. 2. Representación esquemática para ilustrar los parámetros de proceso y los efectos inducidos por el shot peening.

respuesta experimentalmente determinados son los (A) esfuerzos residuales, (B) rugosidad en términos de concentraciones de esfuerzos y (C) endurecimiento por deformación. Con la intención de clarificar los datos que intervienen en el proceso, en la Fig. 2 se ilustra cada uno de los factores y efectos que modifican el estado superficial de un componente.

En los tres factores de control o de procesamiento que se consideraron, se evaluaron cuatro niveles de acción, como se muestra en la Tabla 1. Los factores elegidos son de fundamental importancia para la industria aeroespacial y automotriz [17].

Basándose en los diferentes factores y niveles, y utilizando la metodología diseño de experimentos, se eligió un arreglo ortogonal factorial fraccionado $L_{16}(4)^{5}$. Este enfoque permite alcanzar conclusiones que son válidas en un rango de condiciones experimentales, además de que representa una elección razonable en términos de la reducción del número de experimentos sin pérdida de calidad en la obtención de resultados. El arreglo ortogonal se ilustra en la Tabla 2.

Cabe mencionar que el arreglo ortogonal está construido para usarse con cinco factores, por lo que se necesitó dejar dos columnas vacías.

Esta acción apoyó en forma positiva el diseño experimental porque todos los parámetros que influencian los efectos del proceso son considerados.

Las propiedades de respuesta que resultan de los experimentos de acuerdo con la Tabla 2, se muestran en la Tabla 3. Los esfuerzos residuales se determinaron utilizando el método del agujero ciego, mientras que para el endurecimiento por deformación se 
Alicia Guadalupe Lazcano Herrera, Sandra Silvia Roblero Aguilar, José Solis Romero, et al.

Tabla 1. Factores y sus respectivos niveles de control.

\begin{tabular}{llcccc}
\hline & \multirow{2}{*}{ Factor } & \multicolumn{4}{c}{ Nivel del factor } \\
\cline { 3 - 6 } & & $\mathbf{1}$ & $\mathbf{2}$ & $\mathbf{3}$ & $\mathbf{4}$ \\
\hline $\mathrm{A}$ & Tipo de shot & S230 & CCW20 & S110 & S330 \\
B & Cobertura & 50 & 100 & 200 & 400 \\
D & Ángulo de incidencia & 30 & 90 & 45 & 90 \\
\hline
\end{tabular}

Tabla 2. Arreglo ortogonal para la experimentación.

\begin{tabular}{|c|c|c|c|c|c|c|c|c|c|c|}
\hline \multicolumn{6}{|c|}{ Arreglo ortogonal } & \multicolumn{5}{|c|}{ Factores asignados y sus niveles } \\
\hline Exp. & $\mathbf{A}$ & $\mathbf{B}$ & $\mathbf{C}$ & D & $\mathbf{E}$ & $\begin{array}{l}\text { SHOT } \\
\text { (A) }\end{array}$ & $\begin{array}{c}\text { Cobertura }(\%) \\
\text { (B) }\end{array}$ & $\begin{array}{l}\text { Vacío } \\
\text { (C) }\end{array}$ & $\begin{array}{l}\left.\text { Ángulo ( }{ }^{\circ}\right) \\
\text { (D) }\end{array}$ & $\begin{array}{c}\text { Vacío } \\
\text { (E) }\end{array}$ \\
\hline 1 & 1 & 1 & 1 & 1 & 1 & $\mathrm{~S} 230$ & 50 & - & 30 & - \\
\hline 2 & 1 & 2 & 2 & 2 & 2 & $\mathrm{~S} 230$ & 100 & - & 90 & - \\
\hline 3 & 1 & 3 & 3 & 3 & 3 & $\mathrm{~S} 230$ & 200 & - & 45 & - \\
\hline 4 & 1 & 4 & 4 & 4 & 4 & $\mathrm{~S} 230$ & 400 & - & 90 & - \\
\hline 5 & 2 & 1 & 2 & 3 & 4 & CCW20 & 50 & - & 45 & - \\
\hline 6 & 2 & 2 & 1 & 4 & 3 & CCW20 & 100 & - & 90 & - \\
\hline 7 & 2 & 3 & 4 & 1 & 2 & CCW20 & 200 & - & 30 & - \\
\hline 8 & 2 & 4 & 3 & 2 & 1 & CCW20 & 400 & - & 90 & - \\
\hline 9 & 3 & 1 & 3 & 4 & 2 & S110 & 50 & - & 90 & - \\
\hline 10 & 3 & 2 & 4 & 3 & 1 & S110 & 100 & - & 45 & - \\
\hline 11 & 3 & 3 & 1 & 2 & 4 & $\mathrm{~S} 110$ & 200 & - & 90 & - \\
\hline 12 & 3 & 4 & 2 & 1 & 3 & S110 & 400 & - & 30 & - \\
\hline 13 & 4 & 1 & 4 & 2 & 3 & S330 & 50 & - & 90 & - \\
\hline 14 & 4 & 2 & 3 & 1 & 4 & S330 & 100 & - & 30 & - \\
\hline 15 & 4 & 3 & 2 & 4 & 1 & S330 & 200 & - & 90 & - \\
\hline 16 & 4 & 4 & 1 & 3 & 2 & S330 & 400 & - & 45 & - \\
\hline
\end{tabular}

usó un durómetro Vickers y para la concentración de esfuerzos se consideró un perfilómetro. Para los detalles específicos, se puede consultar [18]. 
Tabla 3. Propiedades de respuesta experimental.

\begin{tabular}{cccc}
\hline No. Exp. & $\begin{array}{c}\text { Esfuerzos } \\
\text { residuales } \\
\text { (Mega Pascales) }\end{array}$ & $\begin{array}{c}\text { Endurecimiento } \\
\text { (Dureza Vickers) }\end{array}$ & $\begin{array}{c}\text { Concentración de } \\
\text { esfuerzos } \\
\text { (adimensional) }\end{array}$ \\
\hline 1 & ER & ED & CE \\
\hline 2 & -124.80 & 144.61 & 1.36 \\
\hline 3 & -233.70 & 145.15 & 1.55 \\
\hline 4 & -197.20 & 154.74 & 1.66 \\
\hline 5 & -246.65 & 166.90 & 1.62 \\
\hline 6 & -173.15 & 135.65 & 1.56 \\
\hline 7 & -243.00 & 147.45 & 1.72 \\
\hline 8 & -164.95 & 150.08 & 1.63 \\
\hline 9 & -245.55 & 157.55 & 1.84 \\
\hline 10 & -280.00 & 145.26 & 1.58 \\
\hline 11 & -131.50 & 153.50 & 1.57 \\
\hline 12 & -379.20 & 157.50 & 1.53 \\
\hline 13 & -149.37 & 151.75 & 1.43 \\
\hline 14 & -257.90 & 134.50 & 1.57 \\
\hline 15 & -170.00 & 159.00 & \\
\hline 16 & -308.50 & 157.89 & 153 \\
\hline
\end{tabular}

\subsection{Fusificación}

Establecidos los datos, se procede a definir las variables y valores linguiísticos, así como la función de pertenencia para realizar la fusificación. Dos subconjuntos difusos (bajo y alto) se asignaron uniformemente en tres variables de entrada, designadas como: Esfuerzo Residual (ER), Concentración de Esfuerzos (CE) y endurecimiento por deformación (ED). Bajo esta premisa, los valores que se asignaron en términos de pertenencia se compilan en la Tabla 4.

Los valores de los datos de entrada se deben definir como FPs. Una FP puede adoptar diferentes formas: trapezoidal, triangular sigmoidal, gaussiana, o una combinación de formas para cada entrada. Las FPs triangulares y trapezoidales se consideraron para las entradas debido a su frecuencia de uso en aplicaciones de ingeniería, con la intención de encontrar los mejores resultados entre ellas. En la Fig. 3 se representa gráficamente las funciones de pertenencia para las tres entradas, las cuales se generaron por medio del software comercial Matlab.

\subsection{Definición de las reglas difusas}

El método Mandani se eligió como el motor de inferencia, el cual se basa en la colección de reglas de control del tipo si-entonces, $y$ (if-then, and, por su nombre en inglés, respectivamente) las cuales se definieron con la siguiente configuración: 
Alicia Guadalupe Lazcano Herrera, Sandra Silvia Roblero Aguilar, José Solis Romero, et al.

Tabla 4. Funciones de pertenencia de las propiedades respuesta con valores nítidos.

\begin{tabular}{cccc}
\hline $\begin{array}{c}\text { Variable } \\
\text { Lingüística }\end{array}$ & $\begin{array}{c}\text { Valores } \\
\text { Lingǘsticos }\end{array}$ & $\begin{array}{c}\text { Función de pertenencia } \\
\text { Triangular }\end{array}$ & $\begin{array}{c}\text { Función de pertenencia } \\
\text { Trapezoidal }\end{array}$ \\
\hline \multirow{2}{*}{$\mathbf{E R}\left(\boldsymbol{K}_{1}\right)$} & Bajo $\left(L_{1}\right)$ & $\mu_{l_{1}}^{k_{1}}:(-570,-379.2,-188.4)$ & $\mu_{l_{1}}^{k_{1}}:(-570,-379.2,-359.2,-188.4)$ \\
\cline { 2 - 4 } & Alto $\left(L_{2}\right)$ & $\mu_{l_{2}}^{k_{1}}:(-315.6,-124.8,315.6)$ & $\mu_{l_{2}}^{k_{1}}:(-315.6,-144.8,-124.8,315.6)$ \\
\hline \multirow{3}{*}{$\mathbf{E D}\left(\boldsymbol{K}_{2}\right)$} & Bajo $\left(L_{1}\right)$ & $\mu_{l_{1}}^{k_{2}}:(110.2,134.5,158.8)$ & $\mu_{l_{1}}^{k_{2}}:(110.2,134.5,136.5,158.8)$ \\
\cline { 2 - 4 } & Alto $\left(L_{2}\right)$ & $\mu_{l_{2}}^{k_{2}}:(142.6,166.9,191.2)$ & $\mu_{l_{2}}^{k_{1}}:(142.6,164.9,166.9,191.2)$ \\
\hline \multirow{2}{*}{$\mathbf{C E}\left(\boldsymbol{K}_{3}\right)$} & Bajo $\left(L_{1}\right)$ & $\mu_{l_{1}}^{k_{3}}:(1.0,1.36,1.72)$ & $\mu_{l_{1}}^{k_{3}}:(1.0,1.36,1.39,1.72)$ \\
\cline { 2 - 4 } & Alto $\left(L_{2}\right)$ & $\mu_{l_{2}}^{k_{3}}:(1.48,1.84,2.2)$ & $\mu_{l_{2}}^{k_{1}}:(1.48,1.81,1.84,2.2)$ \\
\hline & & &
\end{tabular}

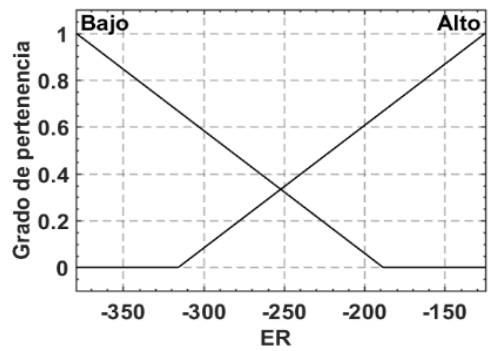

(a)
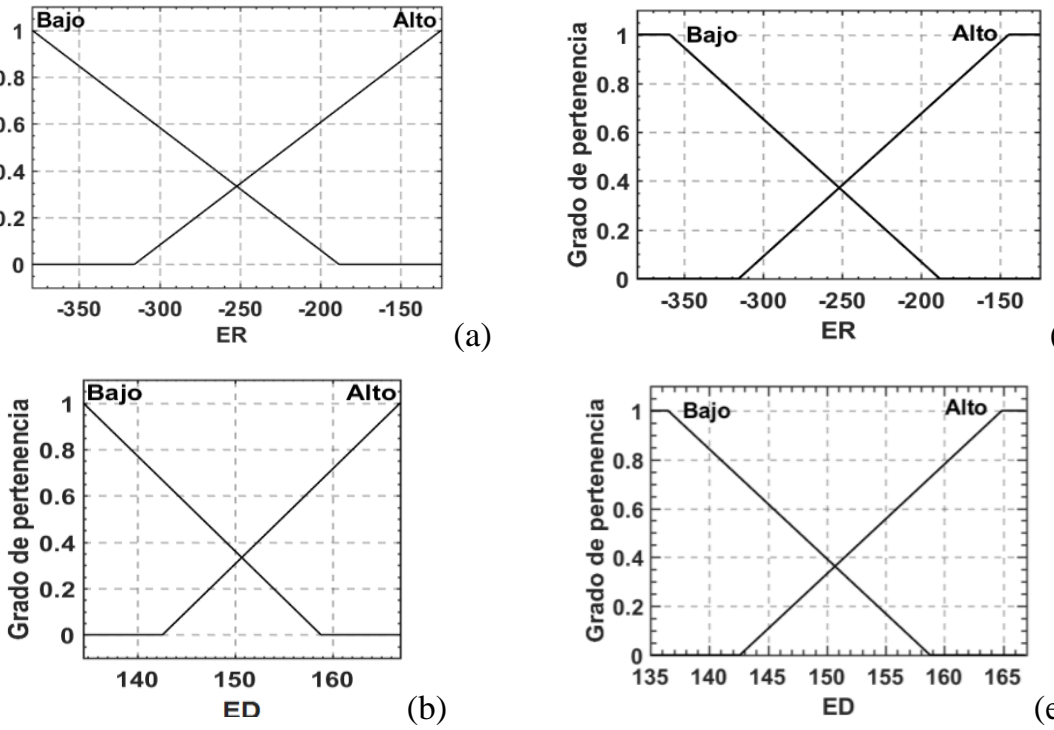

(b)

(e)
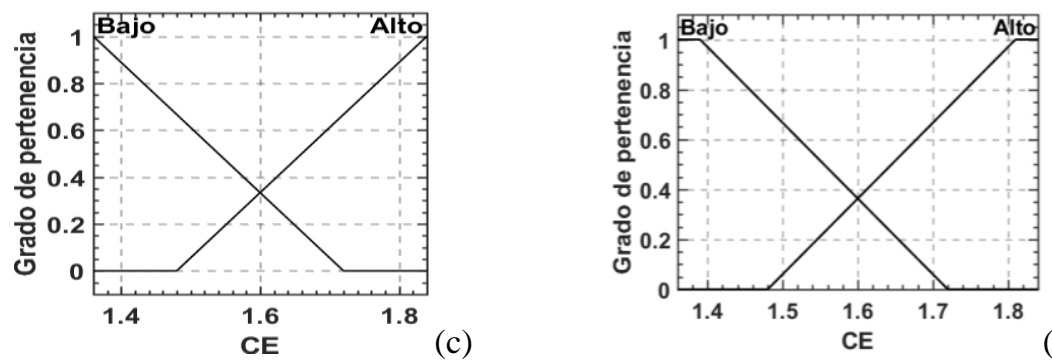

(c)

Fig. 3. Funciones de pertenencia de las propiedades de respuesta como variables de entrada: triangulares (a-c), y trapezoidales (d-f). Triangular (a) y trapezoidal (b).

Regla 1: if ER es Bajo and ED es Bajo and CE es Bajo then IR es MuyBajo Regla 2: if ER es Bajo and ED es Bajo and CE es Alto then IR es Bajo 

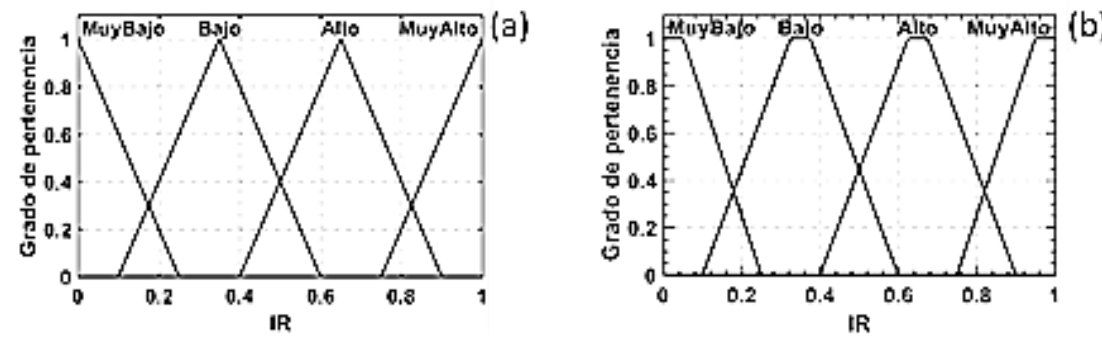

Fig. 4. Funciones de pertenencia de la salida en términos del índice de respuesta (IR).

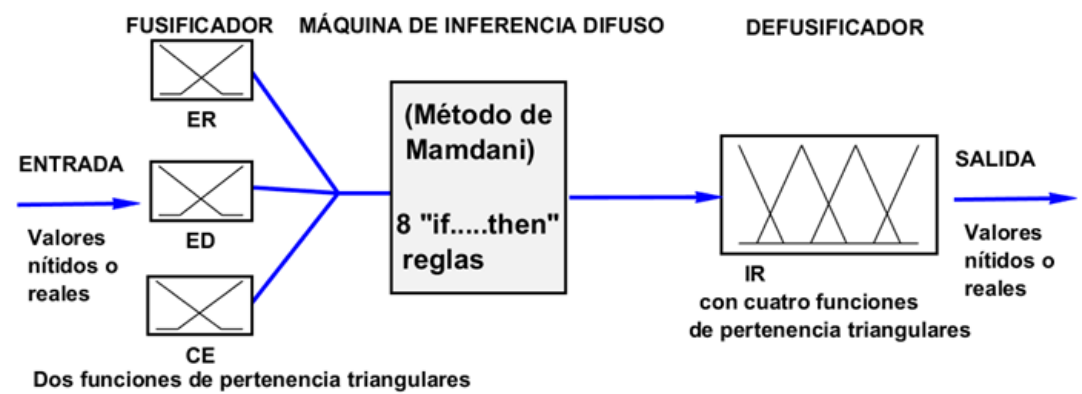

Fig. 5. Representación esquemática de la arquitectura de la unidad de inferencia difusa, ejemplificada con las funciones de pertenencia triangulares.

Regla 3: if ER es Bajo and ED es Alto and CE es Bajo then IR es Bajo Regla 4: if ER es Bajo and ED es Alto and CE es Alto then IR es Alto Regla 5: if ER es Alto and ED es Bajo and CE es Bajo then IR es Bajo Regla 6: if ER es Alto and ED es Bajo and CE es Alto then IR es Alto Regla 7: if ER es Alto and ED es Alto and CE es Bajo then IR es Alto Regla 8: if ER es Alto and ED es Alto and CE es Alto then IR es MuyAlto

\subsection{Aplicación del sistema de inferencia difuso}

Para la defusificación se definió una variable de salida, designada como índice de respuesta (IR), con cuatro valores lingüísticos: Muy Bajo, Bajo, Alto y Muy Alto, como se puede apreciar en la Fig. 4.

Es importante señalar que para la defusificación se emplea el centro de gravedad (COG), lo cual implica que el valor a obtener para el índice IR se ubica en el centro del rango de pertenencia. En la Fig. 5 se muestra el esquema del modelo difuso propuesto descrito en este apartado, tomando como ejemplo un elemento en cada etapa.

\section{Análisis de resultados}

El índice IR determinado de las propiedades multi-respuesta se obtiene utilizando la herramienta de lógica difusa que trabaja con el software comercial Matlab. Los valores 
Alicia Guadalupe Lazcano Herrera, Sandra Silvia Roblero Aguilar, José Solis Romero, et al.

Tabla 5. Salida de FIS mostrando los IRs como valores nítidos.

\begin{tabular}{cccccc}
\hline \multirow{2}{*}{$\begin{array}{c}\text { Exp. } \\
\text { No. }\end{array}$} & \multicolumn{2}{c}{ Valores nítidos de entrada } & \multicolumn{2}{c}{ Valores nítidos de salida (IRs) } \\
\cline { 2 - 6 } & ER & ED & CE & FP:Triangular & FP: Trapezoidal \\
\hline 1 & -124.8 & 144.61 & 1.36 & 0.392 & 0.391 \\
2 & -233.7 & 145.15 & 1.55 & 0.424 & 0.422 \\
3 & -197.2 & 154.74 & 1.66 & 0.647 & 0.65 \\
4 & -246.65 & 166.9 & 1.62 & 0.592 & 0.594 \\
5 & -173.15 & 135.65 & 1.56 & 0.454 & 0.453 \\
6 & -243 & 147.45 & 1.72 & 0.561 & 0.56 \\
7 & -164.95 & 150.08 & 1.63 & 0.593 & 0.593 \\
8 & -245.55 & 157.55 & 1.84 & 0.696 & 0.698 \\
9 & -280 & 145.26 & 1.55 & 0.388 & 0.387 \\
10 & -131.5 & 153.5 & 1.58 & 0.599 & 0.599 \\
11 & -379.2 & 157.5 & 1.57 & 0.455 & 0.455 \\
12 & -149.37 & 151.75 & 1.53 & 0.546 & 0.546 \\
13 & -257.9 & 134.5 & 1.45 & 0.267 & 0.267 \\
14 & -170 & 159 & 1.43 & 0.65 & 0.65 \\
15 & -308.5 & 157.89 & 1.57 & 0.466 & 0.466 \\
16 & -292.3 & 153.58 & 1.54 & 0.423 & 0.424 \\
\hline
\end{tabular}

numéricos nítidos se muestran en la Tabla 5. Evidentemente los valores numéricos de cada función de pertenencia utilizada prácticamente no presentan discrepancia significativa, lo que claramente indica que las formas de pertenencia lineales no influyen en el tratamiento de entradas multi-respuesta a fin de alcanzar un índice de respuesta.

Entre más alto sea el valor del IR más alta la posibilidad de que esa corrida experimental represente la óptima. Al respecto, la corrida experimental No. 8 adopta las mejores características de rendimiento múltiple de entre los 16 experimentos para ambas funciones de pertenencia.

La ortogonalidad hace posible extraer el efecto de cada uno de los parámetros de los datos experimentales a sus diferentes niveles, porque cuando se determina el promedio para un nivel de factor, los otros factores en cada uno de sus niveles toman un número equivalente de veces. Así, el promedio de IR para cada uno de los niveles de los parámetros de prueba, al igual que el promedio total de los IRs se muestra en la Tabla 6.

En la tabla se exhiben valores delta (máx.-mín.), cuyo número más alto es indicativo del nivel de significancia de un factor en particular. Con la intención de visualizar el grado de significancia de cada parámetro sobre los efectos del SP, en la gráfica de la Fig. 6 se muestra el comportamiento de cada parámetro en términos de los promedios IR por nivel. En este caso, inmediatamente se puede deducir que la cobertura tiene la contribución más alta seguida por el shot y al final el ángulo de incidencia. De la misma tabla de respuesta es posible extraer los parámetros que podrían ofrecer la condición óptima para incrementar la resistencia a la fatiga. De acuerdo con la gráfica, la 
Tabla 6. Valores promedio IRs para cada nivel de cada factor.

\begin{tabular}{cccc}
\hline Nivel & Shot & Cobertura & $\begin{array}{c}\text { Ángulo de } \\
\text { incidencia }\end{array}$ \\
\hline 1 & 0.51375 & 0.37525 & 0.54525 \\
\hline 2 & 0.576 & 0.5585 & 0.4605 \\
\hline 3 & 0.497 & 0.54025 & 0.53075 \\
\hline 4 & 0.4515 & 0.56425 & 0.50175 \\
\hline Max-min & 0.1245 & 0.189 & 0.08475 \\
\hline
\end{tabular}

Promedio total de $\mathbf{I R}=0.510$

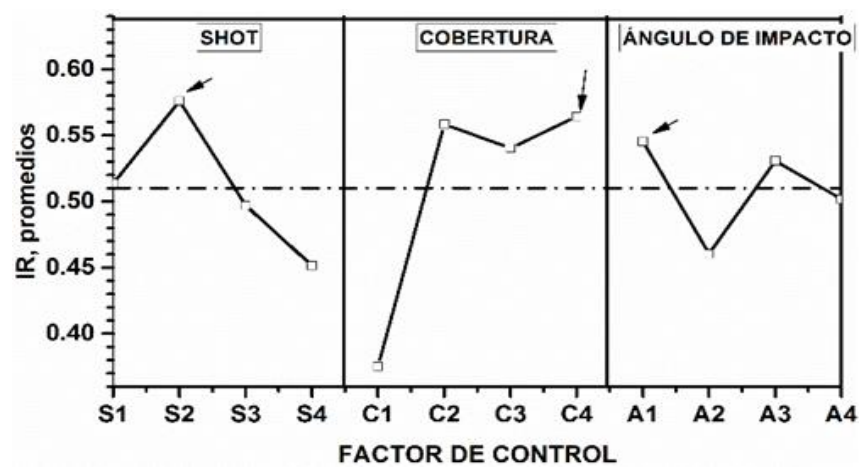

Fig. 6. Factores de control promedio con sus respectivos niveles en términos del IR.

combinación óptima de parámetros, señalada por las flechas, corresponde a S2, C4 y $\mathrm{A} 1$, es decir, de la Tabla 1 , shot $(\mathrm{A} 2)=\mathrm{CW} 20$, cobertura $(\mathrm{B} 4)=400 \%$ y ángulo de incidencia $(\mathrm{D} 1)=30^{\circ}$. Como se esperaba, este resultado confirma el que se determinó en la Tabla 5, resaltando el experimento 8 como el óptimo, el cual tiene el mismo orden de factores y niveles.

\subsection{Pruebas de confirmación}

Toda vez que se determinó la combinación óptima de parámetros de procesamiento con base a los efectos que provoca el SP considerados aquí como la multi-respuesta o tres funciones objetivo, se procede a realizar la comparación con la corrida experimental inicial. Los resultados se presentan en la Tabla 7.

Los resultados experimentales exhiben una ligera ganancia en los esfuerzos residuales (ER), quedando prácticamente el mismo nivel de endurecimiento por deformación (ED), pero con un incremento en concentración de esfuerzos (CE) en las condiciones iniciales respecto a la combinación óptima. Este último resultado repercute negativamente en la resistencia a la fatiga, lo que significa que las condiciones óptimas efectivamente resultaron en una mejora significativa, como se puede apreciar en la Fig. 7. Puede observarse, por ejemplo, que, a $300 \mathrm{MPa}$ de esfuerzo aplicado, la probeta sin tratamiento de SP se fractura (falla) por los 150000 ciclos, entre tanto, la probeta con 
Alicia Guadalupe Lazcano Herrera, Sandra Silvia Roblero Aguilar, José Solis Romero, et al.

Tabla 7. Resultados experimentales bajo condiciones iniciales y óptimas.

\begin{tabular}{|c|c|c|c|c|}
\hline Efectos & $\begin{array}{c}\text { Combinación } \\
\text { óptima } \\
\end{array}$ & $\begin{array}{c}\text { Resultados } \\
\text { experimentales }\end{array}$ & $\begin{array}{c}\text { Condiciones } \\
\text { iniciales } \\
\end{array}$ & $\begin{array}{c}\text { Resultados } \\
\text { experimentales }\end{array}$ \\
\hline ER & \multirow{3}{*}{$\begin{array}{c}\mathrm{A} 2, \mathrm{~B} 4, \mathrm{D} 1 \\
\left.\mathrm{~W} 20,400 \%, 30^{\circ}\right)\end{array}$} & $300 \mathrm{MPa}$ & \multirow{3}{*}{$\begin{array}{c}\mathrm{A} 4, \mathrm{~B} 4, \mathrm{D} 4 \\
\left(\mathrm{~S} 330,400 \%, 90^{\circ}\right)\end{array}$} & $373 \mathrm{MPa}$ \\
\hline ED & & $147 \mathrm{HV}$ & & $150 \mathrm{HV}$ \\
\hline $\mathrm{CE}$ & & 1.3 & & 1.8 \\
\hline
\end{tabular}

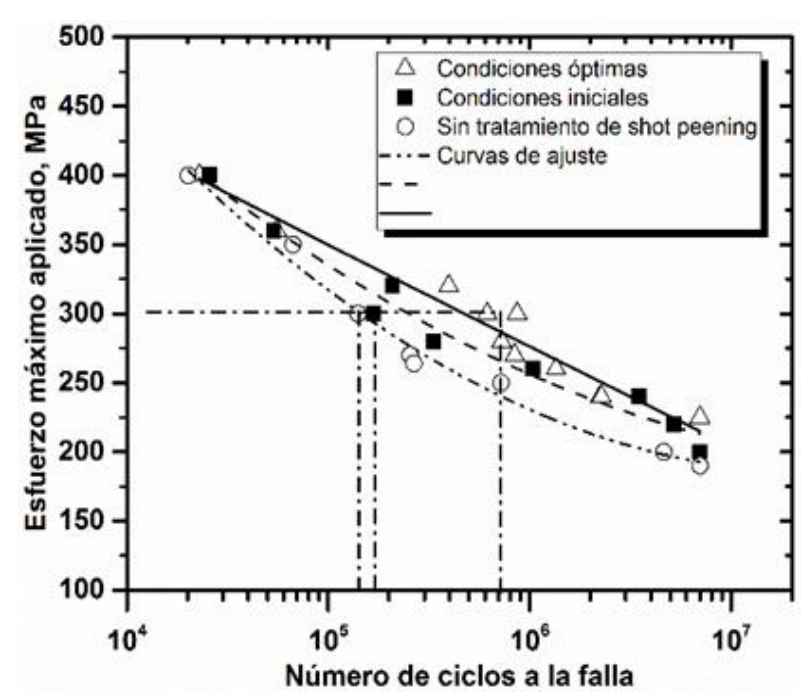

Fig. 7. Curvas S-N, bajo carga axial y amplitud constante para probetas con SP y sin SP.

las condiciones iniciales ( la que se aplicó tratamiento empleando las condiciones determinadas con lógica difusa se fracturó por los 700000 ciclos. La resistencia a la falla por fatiga se incrementó hasta por un $300 \%$ con respecto a las condiciones iniciales.

\section{Conclusiones y trabajo futuro}

En relación con los resultados alcanzados en las pruebas de confirmación, se pueden configurar las siguientes conclusiones:

- El modelo de inferencia difuso es efectivo para tratar con aplicaciones ingenieriles multi-respuesta, en forma sencilla y con bajo costo. El proceso de shot peening, así como otros procesos de la ingeniería de superficies (como recubrimientos) son factibles para implementar el uso de la inteligencia artificial.

- En el presente estudio, el proceso de SP, se determinó una combinación de factores de control, mediante los cuales se generaron las pruebas de fatiga que 
resultaron con un incremento apreciable en la resistencia a la fatiga del aluminio 2024-T351, comúnmente utilizado en estructuras de aeronaves.

- La metodología asociada entre el diseño de experimentos y lógica difusa ofrece una alternativa positiva en la reducción de tiempo y costo en las investigaciones que tratan con resultados no lineales ya que evita el típico trato de prueba y error, así como la realización de una gran cantidad de experimentos que no ofrecen mejores resultados.

- Las funciones de pertenencia lineales no presentan discrepancia significativa cuando se trabaja con respuestas multi-objetivo, por lo que resulta indistinto utilizar la función de pertenencia triangular o trapezoidal para este tipo de aplicaciones.

La aplicación de la técnica de cómputo suave permitió integrar tres respuestas con el resultado favorable de una sola salida, lo que posibilitó la elección de la mejor combinación de parámetros de proceso, dado que se manifestó positivamente en los resultados de fatiga. No obstante, es evidente la necesidad de realizar trabajo adicional utilizando técnicas de inteligencia artificial alternativas, por ejemplo: redes neuronales, algoritmos genéticos o una combinación de ellas, con la finalidad de comparativamente establecer resultados en forma individual por cada técnica o híbridos, para no únicamente cubrir aspectos como en la presente aplicación, sino extender la posibilidad de explorar aplicaciones en otras áreas de ingeniería.

\section{Referencias}

1. Suresh, S.: Fatigue of Materials. Second ed. Cambridge, University Press (1998)

2. Miller, K.J.: Materials science perspective of metal fatigue resistance. Materials Science and Technology. 9(6), pp. 453-462 (1993)

3. Chadwick, D.J., Ghanbari, S., Bahr, D.F., Sangid, M.D.: Crack incubation in shot peened AA7050 and mechanism for fatigue enhancement. Fatigue and Fracture of Engineering Materials and Structures, 41(1), pp. 71-83 (2018)

4. Croccolo, D., Cristofolini, L., Bandini, M., Freddi, A.: Fatigue strength of shot-peened nitrided steel: optimization of process parameters by means of design of the experiment. Fatigue and Fracture of Engineering Materials and Structures. 25(7), pp. 695 (2002)

5. Khany, S.E., Moyeed, M.A., Siddiqui, M.S., Ahmed, G.M.S., Baig, M.M.A.: An Experimental Study of the Effect of Shot Peening on the Low Carbon Steel and Identification of Optimal Process Parameters. Materials Today: Proceedings, 2(4), pp. 3363-3370 (2015)

6. Unal, O.: Optimization of shot peening parameters by response surface methodology. Surface and Coatings Technology, 305, pp. 99-109 (2016)

7. George, P.M., Pillai, N., Shah, N.: Optimization of shot peening parameters using Taguchi technique. Journal of Materials Processing Technology, pp. 153-154, pp. 925-930 (2004)

8. Zadeh, L.A.: Toward a theory of fuzzy information granulation and its centrality in human reasoning and fuzzy logic. Fuzzy Sets and Systems, 90(2), pp. 111-127 (1997) 
9. Lin, J.L., Wang, K.S., Yan, B.H., Tarng, Y.S.: Optimization of the electrical discharge machining process based on the Taguchi method with fuzzy logics. Journal of Materials Processing Technology, 102(1), pp. 48-55 (2000)

10. Gupta, A., Singh, H., Aggarwal, A.: Taguchi-fuzzy multi output optimization (MOO) in high speed CNC turning of AISI P-20 tool steel. Expert Systems with Applications, 38(6), pp. 6822-6828 (2011)

11. Kamble, P.D., Waghmare, A.C., Askhedkar, R.D., Sahare, S.B.: Multi Objective Optimization of Turning AISI 4340 Steel Considering Spindle Vibration Using TaguchiFuzzy Inference System. Materials Today: Proceedings, 2(4), pp. 3318-3326 (2015)

12. Moayyedian, M., Abhary, K., Marian, R.: Optimization of injection molding process based on fuzzy quality evaluation and Taguchi experimental design. CIRP Journal of Manufacturing Science and Technology (2018)

13. Zadeh, L.: Fuzzy sets. Information and Control, 8, pp. 338-353 (1965)

14. Dubois, D., Prade, H.: Fuzzy sets and systems: Theory and Applications. Academic Press (1980)

15. Kosko, B.: Fuzzy systems as universal approximators. 43(11), pp. 1329-1333 (1994)

16. Mandani: Application of fuzzy logic to approximate reasoning using linguistic synthesis, IEEE Transactions on Computers (1977)

17. Nam, Y.S., Jeon, U., Yoon, H.K., Shin, B.C., Byun, J.H.: Use of response surface methodology for shot peening process optimization of an aircraft structural part. The International Journal of Advanced Manufacturing Technology, 87(9), pp. 29672981 (2016)

18. Rodopoulos, C.A., Curtis, S.A., De los Rios, E.R., Solis-Romero, J.: Optimisation of the fatigue resistance of 2024-T351 aluminium alloys by controlled shot peeningmethodology, results and analysis. International Journal of Fatigue, 26(8), pp. 849856 (2004) 